\title{
Ginger Seeding Detection and Shoot Orientation Discrimination Using an Improved YOLOv4-LITE Network
}

\author{
Lifa Fang ${ }^{1,2}$, Yanqiang Wu ${ }^{2,3}$, Yuhua Li ${ }^{2,3}$, Hongen Guo ${ }^{1}$, Hua Zhang ${ }^{1}$, Xiaoyu Wang ${ }^{1}$, Rui Xi ${ }^{2,3}$ \\ and Jialin Hou 1,2,* \\ 1 Shandong Academy of Agricultural Machinery Sciences, Jinan 250100, China; 2019110093@sdau.edu.cn (L.F.); \\ 2021110430@sdau.edu.cn (H.G.); 2021110420@sdau.edu.cn (H.Z.); 2018010024@sdau.edu.cn (X.W.) \\ 2 College of Mechanical \& Electronic Engineering, Shandong Agricultural University, Tai'an 271018, China; \\ wuyq@sdau.edu.cn (Y.W.); liyuhua@sdau.edu.cn (Y.L.); xirui@sdau.edu.cn (R.X.) \\ 3 Shandong Agricultural Equipment Intelligent Engineering Laboratory, Tai'an 271018, China \\ * Correspondence: jlhou@sdau.edu.cn; Tel.: +86-538-824-6121
}

check for

updates

Citation: Fang, L.; Wu, Y.; Li, Y.; Guo, H.; Zhang, H.; Wang, X.; Xi, R.; Hou, J. Ginger Seeding Detection and Shoot Orientation Discrimination Using an Improved YOLOv4-LITE Network. Agronomy 2021, 11, 2328. https:// doi.org/10.3390/agronomy11112328

Academic Editors: Saeid Homayouni, Yacine Bouroubi and Karem Chokmani

Received: 16 October 2021

Accepted: 15 November 2021

Published: 17 November 2021

Publisher's Note: MDPI stays neutral with regard to jurisdictional claims in published maps and institutional affiliations.

Copyright: (c) 2021 by the authors. Licensee MDPI, Basel, Switzerland. This article is an open access article distributed under the terms and conditions of the Creative Commons Attribution (CC BY) license (https:// creativecommons.org/licenses/by/ $4.0 /)$.

\begin{abstract}
A consistent orientation of ginger shoots when sowing ginger is more conducive to high yields and later harvesting. However, current ginger sowing mainly relies on manual methods, seriously hindering the ginger industry's development. Existing ginger seeders still require manual assistance in placing ginger seeds to achieve consistent ginger shoot orientation. To address the problem that existing ginger seeders have difficulty in automating seeding and ensuring consistent ginger shoot orientation, this study applies object detection techniques in deep learning to the detection of ginger and proposes a ginger recognition network based on YOLOv4-LITE, which, first, uses MobileNetv2 as the backbone network of the model and, second, adds coordinate attention to MobileNetv2 and uses Do-Conv convolution to replace part of the traditional convolution. After completing the prediction of ginger and ginger shoots, this paper determines ginger shoot orientation by calculating the relative positions of the largest ginger shoot and the ginger. The mean average precision, Params, and giga Flops of the proposed YOLOv4-LITE in the test set reached 98.73\%, $47.99 \mathrm{M}$, and 8.74, respectively. The experimental results show that YOLOv4-LITE achieved ginger seed detection and ginger shoot orientation calculation, and that it provides a technical guarantee for automated ginger seeding.
\end{abstract}

Keywords: image recognition; deep learning; YOLO; attention mechanism; MobileNetv2; ginger

\section{Introduction}

Ginger is a perennial herb whose roots are often made into spices and herbs [1,2]. It originated in Asia and is now widely grown in various regions, of which China is the world's most productive country for ginger [3,4]. Before sowing, growers need to break the ginger and cultivate the ginger shoots, thus retaining one to two ginger shoots for each ginger seed [5]. After plowing, fertilizing, and trenching, the ginger seeds are placed in a trench [6,7], while ensuring consistent ginger shoot orientation. In China, ginger shoots generally face southwest, which is beneficial for ginger shoots to accumulate temperature. This is because ginger is a crop with a high cumulative temperature, and effective temperature accumulation is more conducive to high ginger production [8]. Furthermore, consistent ginger shoot orientation ensures that all gingers grow parallel to each other, effectively avoiding neighboring ginger seeds crowding together and, thus, affecting the quality and yield of ginger. Ginger sowing mainly relies on manual labor [9], while mechanical sowing is less prevalent and still requires manual assistance. At present, ginger mechanical seeding is commonly realized in the following way: growers first place the ginger seeds in the ginger seed holding device and then make them fall into the trench with the help of different forms of conveying device. 
Ginger seeds are treated with medicine to make the color features of the ginger shoots very unstable before sowing, and traditional object detection methods are unable to meet the requirements of rapid and accurate identification. Object detection technology has made remarkable breakthroughs and has been widely applied in the agricultural field with the rapid development of deep learning technology in recent years [10-14]. The main task of object detection is to find out the class and location of targets of interest in an image, and object detection is mainly divided into two categories: one-stage algorithms and two-stage algorithms. Two-stage algorithms require, first, forming a regional proposal, and then the object is classified and localized using convolutional neural networks (CNN). Typical algorithms are Faster-RCNN [15-18], Mask R-CNN [19], etc. Their recognition speed is slow due to the requirement of multiple detection and classification. On the other hand, the one-stage recognition speed is relatively fast, so it is more suitable for mobile applications, because it can directly predict the category and location of objects through features extracted from the network. Commonly used algorithms include YOLOv3 (you only look once v3) [20-22], YOLOv4 [23,24], SSD [25], Retina-Net [26], etc. Hou et al. [27] proposed a ginger shoot identification method based on YOLOv3, but this method only identifies ginger shoots, resulting in a complex process of calculating ginger shoot orientation, and it had a redundant backbone network. The backbone network of YOLO [28] is relatively complex, which makes YOLO unsuitable for direct application in mobile terminals. Therefore, many researchers have put forward different improved methods for the backbone network, significantly reducing the model size, while ensuring the accuracy remains largely unchanged. For instance, they combined YOLOv3 with MobileNetv1 to achieve detection of fish [29], strawberries [30], and citrus fruit [31]; YOLOv4 with MobileNetv3-Small to achieve detection of weeds [32]; YOLOv3 with Darknet19 to achieve detection of mangoes [33], coffee fruits [34], and leaves [35]; and YOLOv4 with CSPDarknet19 to achieve detection of pear fruits [36].

Recently, the attention mechanism has been widely applied in CNN. In the human visual system, people usually selectively focus on the parts they are interested in, rather than the whole scene. Similarly, the purpose of the attention mechanism in neural networks is to filter out the most critical information for the current task from a large number of messages. The common attention mechanisms are squeeze and congestion (SE) attention [37] and convolution block attention module (CBAM) attention [38]. The aforementioned attention mechanisms have been applied in different networks. For illustration, Kang et al. [39] added CBAM attention to an Xception network for segmentation of cotton roots; $\mathrm{Xu}$ et al. [40] applied SE attention for classification of fish in a ResNet network; Yang et al. [41] added CBAM attention to the backbone network of YOLOv4 for detection of wheat ears; Tang et al. [42] implemented the classification of grape leaves by adding SE attention to ShuffleNetv1 and v2; and Li et al. [43] implemented the detection of lemons by adding SE attention to the backbone network of YOLOv3. In addition, one-stage object detection usually suffers from an imbalance between positive and negative samples, which is generally solved by adding different weights to them when calculating the loss function. There are various common methods, such as hard negative mining, online hard negative mining (OHNM), class balance loss, focal loss [26], etc. Among them, focal loss has been the most widely used. For example, Liu et al. [44] combined a YOLO network and focal loss to recognize broken maize, and Li et al. [45] realized the detection of hydroponic lettuce seedings status by combining focal loss and Faster R-CNN.

Based on the above research, this paper proposes a deep learning method based on YOLOv4 to implement the recognition of ginger seeds, and which offers improvements to address some of the problems in the recognition process. First, the YOLOv4 backbone network is very complex and contains many redundant parameters, making it difficult to deploy on the mobile terminals of the ginger planter; therefore, we propose the use of a MobileNetv2 [46] network instead of the original CSPDarknet53 network. Second, the YOLOv4 network is poor at recognizing ginger shoots compared to the larger target ginger, and, thus, we introduce a simple and efficient CA (coordinate attention) [47] mechanism. 
In addition, we introduce Do-Conv (depth-wise over-parameterized convolution) [48] to speed up the network's training and facilitate the convergence of the model. Finally, the recognition targets of this network are ginger shoots and ginger, and the difference in recognition difficulty and target size between them is large. Therefore, we introduce focal loss to solve the problems of positive and negative sample imbalance and simple and difficult sample imbalance. The above improvements provide a technical guarantee for the fast and accurate discrimination of the orientation of ginger shoots in ginger seed images. The rest of the paper is organized as follows: Section 2 describes the creation of the dataset and the improvements based on the YOLOv4 network; Section 3 describes the tuning of the model parameters and the experimental validation of the proposed method; and Section 4 describes the conclusions of this work.

\section{Materials and Methods}

\subsection{Data Processing}

\subsubsection{Data Acquisition and Annotation}

This paper uses ginger seed samples collected from a ginger plantation in Anqiu, Shandong, China $\left(36.47847^{\circ} \mathrm{N}, 119.2189^{\circ} \mathrm{E}\right)$ on 25 April 2021. The ginger seeds are of the 'Baby Ginger' variety and were germinated for 15 days. Ginger seed images were captured to accelerate the training and debugging of the ginger seed recognition model, by using the device shown in Figure 1, which includes a CMOS industrial camera, fill light, camera stand, computer, etc. The camera model was a MV-EM1400C manufactured by Micro-vision, with a resolution of $3288 \times 3288$ pixels; the fixed-focus lens was M1620MPW2, the shooting distance was $30 \mathrm{~cm}$, and a total of 500 images in "JPG" file format were stored. In addition, high luminance, regular luminance, and low luminance images were acquired separately to test the model recognition ability, for a total of 100 images. Meanwhile, 500 ginger seed images from a previous study by Hou et al. [27] were used to enrich the ginger dataset, with an image size of $5472 \times 3672$ pixels.

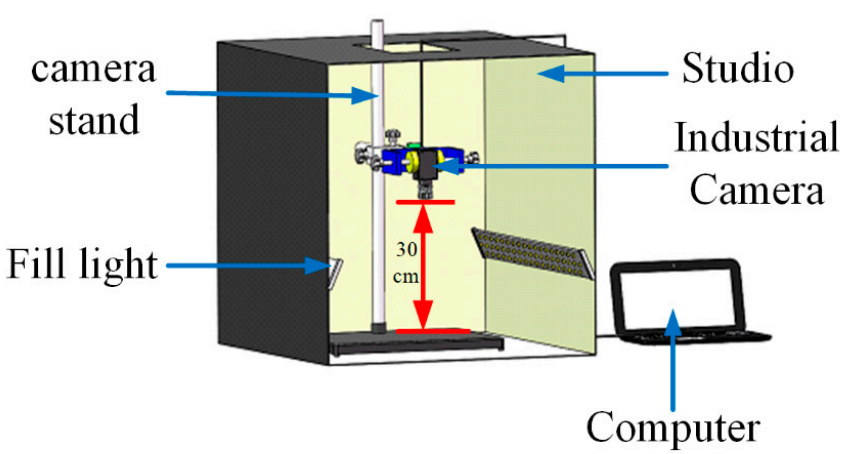

Schematic diagram of the image acquisition

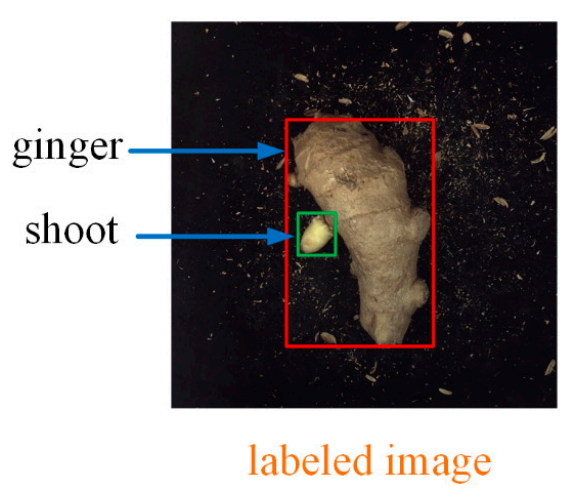

labeled image

/home/lab113/desktop/YoLov4/data/JPEGImages/000195.jpg 1333,2890,2081,3537,0 352,1,4690,2942,1 /home/lab113/desktop/YoLov4/data/JPEGImages/000415.jpg 3428,1918,3881,2442,0 881,799,4143,2257,1 /home/lab113/desktop/YoLov4/data/JPEGImages/060367.jpg 3514,2323,4538,3218,0 24,118,4981,2733,1

Figure 1. Schematic diagram of image acquisition and annotation.

As shown in Figure 1, LabelImg (https://github.com/tzutalin/labelImg, accessed on 9 August 2021) was used to label the ginger shoots and ginger separately in "xml" file format, to determine the orientation of the ginger shoots; and it is worth noting that the labeled boxes are tightly aligned with the edges of the ginger and ginger shoots. In addition,

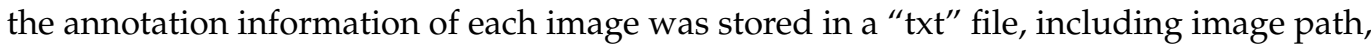
annotation box coordinates (image coordinates of the upper left and lower right corners), and object category. After image annotation, 1000 images were randomly divided into training and validation sets in the ratio of $80 \%$ and $20 \%$, and the remaining 100 images 
were used for the model testing. Among them, the validation set was for adjusting the hyperparameters and monitoring the model for overfitting, and the test set was used for model evaluation, with no duplication between the above two, to ensure the accuracy of the model evaluation results. Finally, the ginger images, annotation files, and category labels were stored in PASCAL VOC format for training the ginger seed recognition network.

\subsubsection{Data Enhancement}

This paper used online data enhancement for expanding the original ginger seed images, to improve the model generalization ability and compensate for the insufficient number of samples. This means that before each batch training, the data-enhanced images were scaled to $416 \times 416$ pixels, and then four images were randomly cropped and stitched into one image using the Mosaic algorithm, thus, serving as training data. Mosaic greatly enriches the image background and also reduces the demand for GPU memory. The data enhancement methods are specified as follows: (1) Horizontal flip, mirror flip, and affine transformation were performed on images, with a 0.5 probability of reducing the effect of different ginger positions on the recognition results. (2) Image brightness was increased by 1.2 times or decreased by 0.8 times, with a 0.5 probability of reducing the effect of different illumination levels on the field on recognition results. (3) Image contrast was increased by 1.2 times or reduced by 0.8 times, with a 0.5 probability of better expressing the grayscale, sharpness, and texture details of the ginger images.

\subsection{Overall Technical Route}

To achieve accurate real-time detection of ginger and ginger seeds, the technical solutions proposed in this study are as follows:

1. Construction and training of a YOLOv4-LITE network. This study used the MobileNetv2 network to replace the original CSPDarknet53, to solve the model redundancy caused by the more complex backbone network.

2. The introduction of an attention mechanism and Do-Conv convolution. This study introduced an attention mechanism and Do-Conv into YOLOv4-LITE, to improve the recognition of smaller ginger shoots.

3. Model performance analysis and experimental validation. The performance of the improved model was tested, and the improvements proposed in this study were verified and analyzed sequentially.

\subsection{Method of Discriminating Ginger Shoot Orientation}

\subsubsection{YOLOv4 Model}

As an end-to-end one-stage object detection algorithm based on regression theory, a YOLO network can directly predict the bounding box and class of an object. The YOLOv4 network is based on the original YOLO and is optimized for data processing, backbone network, activation function, loss function, and other aspects to improve the detection performance and inference speed of the model. Its training process is shown in Figure 2, which includes the following five parts:

1. Based on Darknet53, CSPDarknet53 borrows the cross-stage partial (CSP) from CSPNet and adds a CSP on each of the five residual blocks, which enhances the learning ability of CNN and can maintain a high performance while lowering the weight of the network. CBL (convolution, batch normalization, and Leak-ReLU) is the most common module in YOLOv4 and includes convolutional (Conv) layers, batch normalization layers, and activation layer constructs.

2. This paper adds a spatial pyramid pooling (SPP) structure after CSPDarknet53, which effectively increases the perceptual field of the backbone network. It uses the maximum pooling operations with convolution kernels of $1 \times 1,5 \times 5,9 \times 9$, and $13 \times 13$, respectively, to obtain four feature maps in different scales, and then fuses them in a concatenated manner. 
3. In $\mathrm{CNN}$ networks, shallow features contain richer target location information, such as contours and textures, and less semantic information. However, the deeper features contain richer semantic information, and the object location information is coarse. Therefore, our network adopts a feature pyramid network (FPN) structure, which passes the deep semantic information through up-sampling, thus fusing the shallow layers' semantic information and location information.

4. Borrowing from the bottom-up path augmentation method in PANet [49], two-path aggregation network (PAN) structures are added after FPN, which transmits the underlying location information by down-sampling, thus fusing location information with the semantic information of higher levels.

5. YOLOv4 loss function includes bounding regression loss $\left(L_{\text {coord }}\right)$, based on the complete intersection over union CIoU $\left(L_{\mathrm{CIoU}}\right)$, confidence loss $\left(L_{\text {conf }}\right)$, and classification loss $\left(L_{c l s}\right)$. The loss function is formulated as follows:

$$
\begin{aligned}
\text { Loss } & =L_{\text {coord }}+L_{\text {conf }}+L_{c l s} \\
& =\lambda_{\text {coord }} \sum_{i=0}^{s^{2}} \sum_{j=0}^{B} I_{i j}^{\mathrm{obj}}\left(2-w_{i} * h_{i}\right) L_{C I o U} \\
& +\sum_{i=0}^{s^{2}} \sum_{j=0}^{B} \operatorname{BCE}\left(c_{i}, \hat{c}_{i}\right)\left(I_{i j}^{\mathrm{obj}}+\lambda_{\text {noobj }} I_{i j}^{\mathrm{noobj}}\right) \\
& +\sum_{i=0}^{s^{2}} I_{i j}^{\mathrm{obj}} \sum_{j=0}^{B} \operatorname{BCE}\left(p_{i}, \hat{p}_{i}\right)
\end{aligned}
$$

where $\lambda_{\text {coord }}$ and $\lambda_{\text {noobj }}$ are penalty coefficients; $s^{2}$ is the number of grids in the feature map; $B$ is the number of anchor boxes per grid; $i$ is the $i$-th grid and $j$ is the $j$-th anchor box; $\left(w_{i}, h_{i}\right)$ and $\left(\hat{w}_{i}, \hat{h}_{i}\right)$ are the coordinates of the ground true and the prediction, respectively; $\mathrm{BCE}(\cdot)$ represents the binary cross-entropy loss; $I_{i j}^{\mathrm{obj}}$ and $I_{i j}^{\text {noobj }}$ represent whether there is an object in the $j$-th anchor box of the $i$-th grid, if so, then $I_{i j}^{\mathrm{obj}}=1$ and $I_{i j}^{\text {noobj }}=0$, otherwise, $I_{i j}^{\mathrm{obj}}=0$ and $I_{i j}^{\text {noobj }}=1 ; c_{i}$ and $\hat{c}_{i}$ are the confidence of ground truth and prediction, respectively; $p_{i}$ and $\hat{p}_{i}$ are the probabilities of the output categories of ground truth and prediction, respectively.

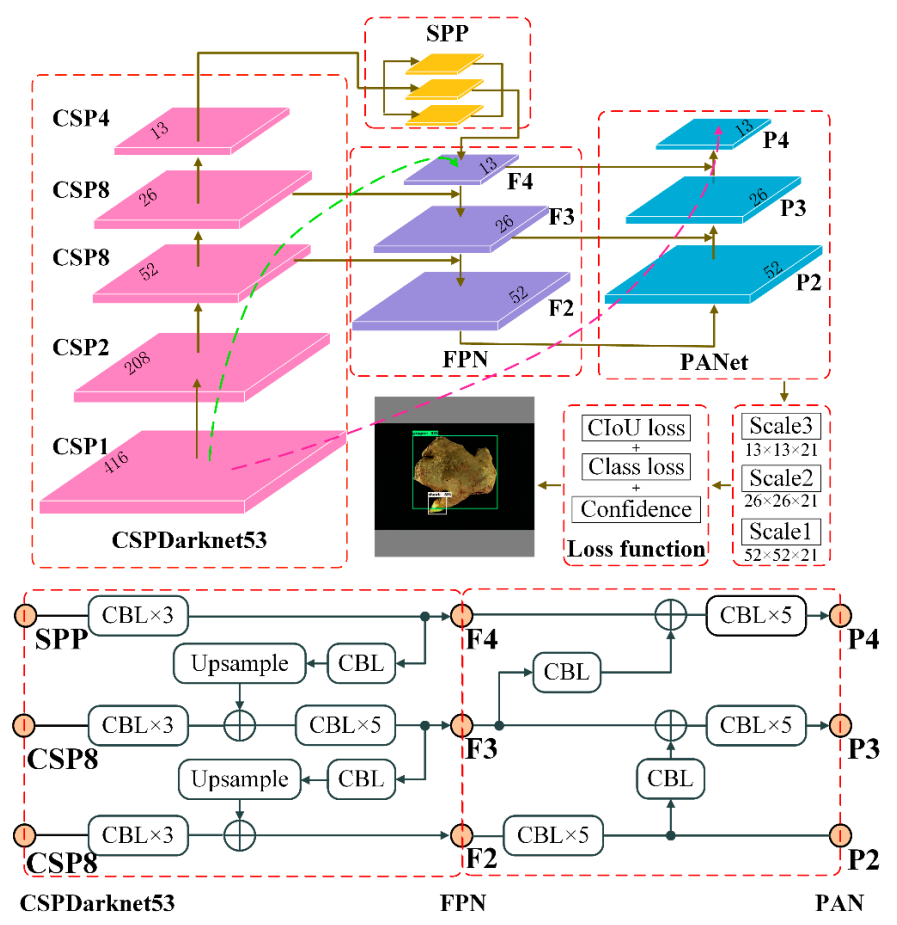

Figure 2. Overview of YOLOv4 network. 


\subsubsection{YOLOv4-LITE Network Design}

Based on the features extracted by the backbone network, the YOLO network predicts object bounding boxes and categories. Moreover, YOLOv4 uses CSPDarknet53, after removing the final pooling layer, fully connected (FC) layer, and softmax layer, as the backbone of the feature extraction network. However, with YOLOv4 it is difficult to achieve a high inference speed in embedded devices, due to its network layer count of 104; requiring a lightweight network to replace the original complex backbone network. Therefore, this paper designed a YOLOv4-LITE network, based on the YOLOv4. In addition, MobileNetv1 is a lightweight network proposed by Google, and it uses depth-wise separable convolution instead of traditional convolution. Hence, this paper used MobileNetv2 as the backbone network of YOLOv4-LITE, which reduces the model size, while maintaining its performance. The network parameters of YOLOv4-LITE are shown in Table 1.

Table 1. YOLOv4-LITE network parameters.

\begin{tabular}{|c|c|c|c|c|c|}
\hline YOLOv4 & No. & Type & Output Size & Stride & Numbers \\
\hline \multirow{10}{*}{ MobileNetv2 } & - & Input & $416 \times 416 \times 3$ & - & - \\
\hline & 0 & CBL & $208 \times 208 \times 32$ & 2 & 1 \\
\hline & $1-4$ & IRB2 & $208 \times 208 \times 16$ & 2 & 1 \\
\hline & $5-11$ & IRB1 & $104 \times 104 \times 24$ & 1 & 2 \\
\hline & $12-22$ & IRB2 & $52 \times 52 \times 32$ & 2 & 3 \\
\hline & $23-37$ & IRB2 & $52 \times 52 \times 64$ & 2 & 4 \\
\hline & $38-49$ & IRB1 & $26 \times 26 \times 96$ & 1 & 3 \\
\hline & $50-60$ & IRB2 & $13 \times 13 \times 160$ & 2 & 3 \\
\hline & $61-64$ & IRB2 & $13 \times 13 \times 320$ & 2 & 1 \\
\hline & 65 & Conv $1 \times 1$ & $13 \times 13 \times 1280$ & 1 & 1 \\
\hline \multirow{2}{*}{ SPP } & $66-68$ & CBL(F4) & $13 \times 13 \times 640$ & 1 & 3 \\
\hline & $69-73$ & Max-pooling & $13 \times 13 \times 640$ & 1 & 3 \\
\hline \multirow{11}{*}{ FPN + PANet } & $74-76$ & CBL & $13 \times 13 \times 640$ & 1 & 3 \\
\hline & 77 & CBL & $13 \times 13 \times 48$ & 1 & 1 \\
\hline & 78 & Up-sample & $26 \times 26 \times 48$ & - & 1 \\
\hline & $79-80$ & Route + CBL & $26 \times 26 \times 48$ & 1 & 1 \\
\hline & 81 & Concatenate & $26 \times 26 \times 96$ & - & 1 \\
\hline & $82-86$ & CBL(F3) & $26 \times 26 \times 48$ & 1 & 5 \\
\hline & 87 & CBL & $26 \times 26 \times 16$ & 1 & 1 \\
\hline & 88 & Up-sample & $52 \times 52 \times 16$ & - & 1 \\
\hline & $89-90$ & Route + CBL & $52 \times 52 \times 16$ & 1 & 1 \\
\hline & 91 & Concatenate(F2) & $52 \times 52 \times 32$ & - & 1 \\
\hline & $92-96$ & $\mathrm{CBL}(\mathrm{P} 2)$ & $52 \times 52 \times 16$ & 1 & 5 \\
\hline \multirow{3}{*}{ Head } & 97 & CBL & $52 \times 52 \times 32$ & 1 & 1 \\
\hline & 98 & Conv $1 \times 1$ & $52 \times 52 \times 21$ & 1 & 1 \\
\hline & 99 & Detection & - & - & 1 \\
\hline \multirow{3}{*}{ FPN + PANet } & $100-101$ & Route + CBL & $26 \times 26 \times 48$ & 2 & 1 \\
\hline & 102 & Concatenate & $26 \times 26 \times 96$ & - & 1 \\
\hline & 103-107 & CBL(P3) & $26 \times 26 \times 48$ & 1 & 5 \\
\hline \multirow{3}{*}{ Head } & 108 & CBL & $26 \times 26 \times 96$ & 1 & 1 \\
\hline & 109 & Conv $1 \times 1$ & $26 \times 26 \times 21$ & 1 & 1 \\
\hline & 110 & Detection & - & - & 1 \\
\hline \multirow{3}{*}{ FPN + PANet } & $111-112$ & Route + CBL & $13 \times 13 \times 640$ & 2 & 1 \\
\hline & 113 & Concatenate & $13 \times 13 \times 1280$ & - & 1 \\
\hline & 114-118 & CBL(P4) & $13 \times 13 \times 640$ & 1 & 5 \\
\hline \multirow{3}{*}{ Head } & 119 & CBL & $13 \times 13 \times 1280$ & 1 & 1 \\
\hline & 120 & Conv & $13 \times 13 \times 21$ & 1 & 1 \\
\hline & 121 & Detection & - & - & 1 \\
\hline
\end{tabular}

As can be seen from Table 1, MobileNetv2 mainly consists of two forms of inverse residual block (IRB) that use depth-wise (DW) convolution and point-wise (PW) convolution to extract the image depth features, thus, greatly reducing the time complexity and space complexity of the convolution operations. Figure 3 is a schematic diagram of the improved backbone network. As shown in Figure 3, when stride $=1$ in DW convolution, the block is the inverse residual block $1\left(\mathrm{IRB}_{1}\right)$; when stride $=2$ in DW convolution, the 
block is the inverse residual block $2\left(\mathrm{IRB}_{2}\right)$. Since the convolutional layer in $\mathrm{IRB}_{2}$ also has a down-sampling function, shortcut is not used to keep the output dimension consistent. The above two kinds of IRBs are composed of $\mathrm{PW}_{1}$ convolution, $\mathrm{DW}$ convolution, and $\mathrm{PW}_{2}$ convolution. Among them, $\mathrm{PW}_{1}$ convolution consists of $1 \times 1$ convolution, BN, and ReLU6, and it maps the feature dimension, from low-dimensional space to high-dimensional space, which is beneficial for feature extraction; DW convolution is composed of $3 \times 3$ convolution, $\mathrm{BN}$, and ReLU6, and realizes feature extraction; the $\mathrm{PW}_{2}$ convolution is composed of $1 \times 1$ convolution and $\mathrm{BN}$ to map the high-dimensional space to the low-dimensional space. As the ReLU6 activation function would destroy the features learned by the CNN in the low-dimensional space, the $\mathrm{PW}_{2}$ convolution is not followed by an activation function.

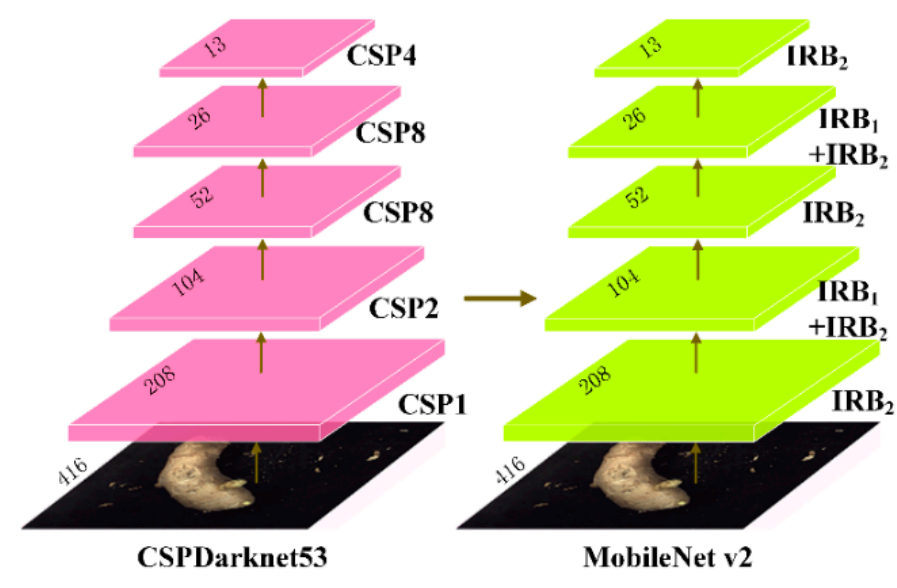

Figure 3. Improved backbone network.

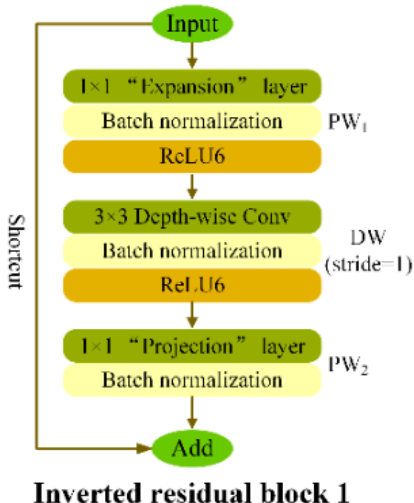

$\left(\right.$ IRB $\left._{1}\right)$

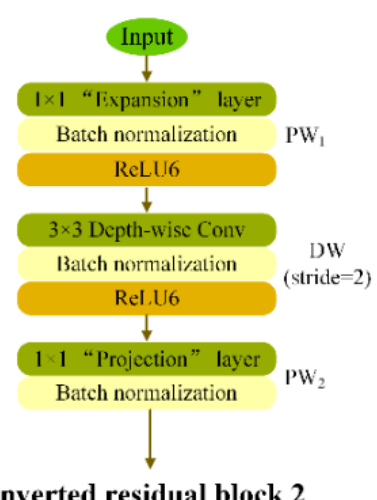

$\left(\mathrm{IRB}_{2}\right)$

\subsubsection{Coordinate Attention Module}

In this paper, an attention [50] mechanism is introduced to improve the model accuracy, by selecting the most critical information for the current recognition task from a large amount of feature information. This is essentially similar to human selective vision, in that it quickly scans the global image to obtain the information that needs to be focused on, while suppressing information that is not helpful for the current task. Therefore, the attention mechanism is applied after DW convolution in the IRB of MobileNetv2. Figure 4 shows the schematic diagram of different attention mechanisms, with an input feature map of size: Height $(H) \times$ Width $(W) \times$ Channel $(C)$.

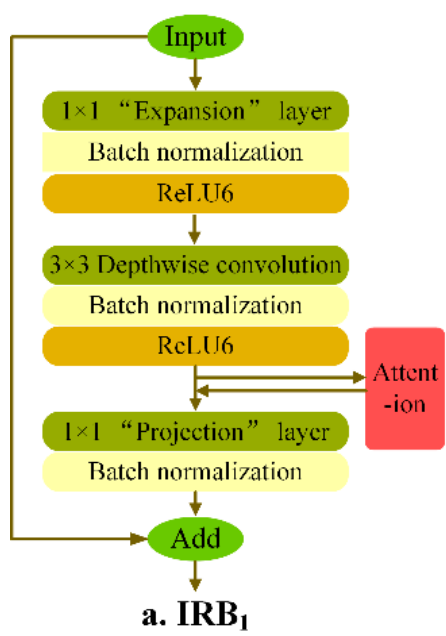

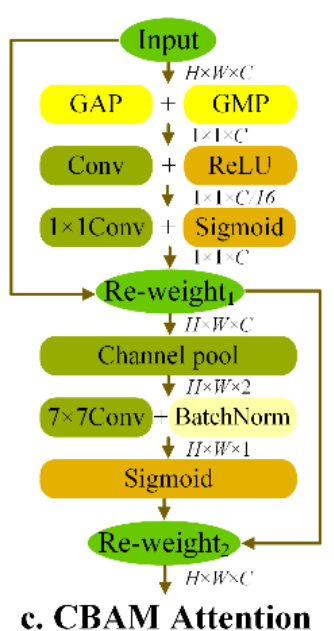

c. CBAM Attention

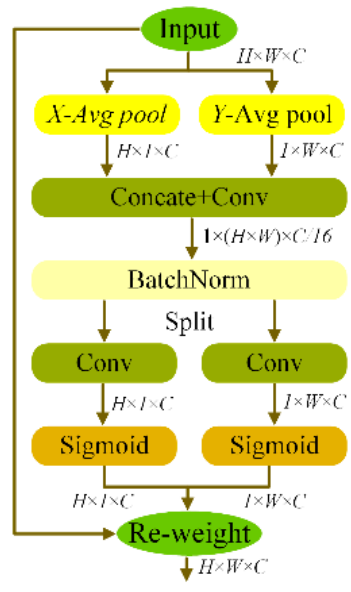

d. CA Attention

Figure 4. Diagram of different attention mechanisms. (a) IRB $;$; (b) SE Attention; (c) CBAM Attention; (d) CA Attention. 
Figure $4 \mathrm{~b}$ shows a schematic structure of SE attention. As the convolution operation only integrates the information of the spatial dimension and channel dimension within a local perceptual field, it does not obtain enough information between global channels. Therefore, first, global average pooling (GAP) is used to compress feature $1(H \times W \times C)$, which has global spatial information, into feature $2(1 \times 1 \times C)$, which has a global receptive field. Second, two FC layers are used to reduce the complexity and improve the generalization ability of the network; where the first FC is used to reduce the dimensionality of the feature map, and the second FC is used to recover the feature dimensionality. Third, feature $3(1 \times 1 \times C)$ is obtained after the sigmoid activation function, which characterizes the importance of each channel. Finally, the channels of feature 3 are multiplied one by one with feature map 1 to obtain the final output re-weight $(H \times W \times C)$, which is equivalent to adding a weight to each channel of feature 1 , thus giving greater weight to information helpful for the task at hand. In conclusion, SE attention improves the sensitivity of the network to channel features and contributes a performance improvement by lowering the computation needed, but it ignores the importance of the location feature information.

Figure $4 \mathrm{c}$ shows a schematic structure of CBAM attention, including the channel attention module (CAM) and spatial attention module (SAM). On the one hand, CAM is similar to SE attention, in that it first compresses the input feature $1(H \times W \times C)$ into feature $2(1 \times 1 \times C)$ using GAP and global max pooling (GMP), which adds a layer of GMP with respect to SE attention; thus, increasing the feature dimension once again. Second, feature 2 is first reduced in the channel dimension to $C / 16$ using a convolution layer, and then its dimension is raised using $1 \times 1$ convolution to obtain feature $3(1 \times 1 \times C)$. Third, feature $4(H \times W \times 1)$ is gained after the sigmoid activation function, which characterizes the importance of the channel feature information. Finally, the Re-weight $1(H \times W \times C)$ is obtained by multiplying feature 4 with feature 1 . On the other hand, in the SAM, first, Re-weight 1 is compressed into feature $5(H \times W \times 1)$ and features $6(H \times W \times 1)$ along the channel direction, using GAP and GMP, respectively, and then they are concatenated based on the channel direction to obtain feature $7(H \times W \times 2)$. Second, the channel dimension of feature 7 is reduced to 1 using $7 \times 7$ convolution, resulting in feature $8(H \times W \times 1)$. Third, feature $9(H \times W \times 1)$ is gained after sigmoid activation, and this characterizes the importance of the location feature information. Finally, the final output Re-weight $2(H \times W$ $\times C$ ) is obtained by multiplying feature 9 with Re-weight ${ }_{1}$, which is equivalent to adding a weight to the location features of Re-weight ${ }_{1}$, so that the location helpful information for the current task has greater weight.

The above two attention mechanisms are widely used in lightweight networks and have achieved good results. However, SE attention only considers the channel feature information and ignores the location information, and CBAM attention only introduces local location information through global pooling. Therefore, this paper presents a coordination attention mechanism, in which location information is embedded into channel attention to avoid adding a large amount of additional computational overheads, while ensuring better attention results for MobileNetv2. Figure $4 \mathrm{~d}$ shows a schematic structure of CA attention, consisting of coordinate information embedding and coordinate attention generation. Each channel is first encoded along two spatial directions, vertical and horizontal, using GAP with pooling kernel sizes $(H, 1)$ and $(1, W)$, respectively, to avoid a possible loss of valuable location information by global pooling in channel attention. The above enables the input feature map $1(H \times W \times C)$ to be compressed into a pair of direction-aware features, including feature $2(H \times 1 \times C)$ and feature $3(1 \times W \times C)$, and they have global receptive field and precise location information. Second, feature $4(1 \times(H+W) \times(C / 16))$ is obtained after concatenating feature 2 with 3 and reducing the feature dimension using $1 \times 1$ convolution. Then, feature 4 is decomposed into feature 5, 6 along the spatial dimension, and their feature dimensions are elevated using $1 \times 1$ convolution, and the above operation greatly reduces the model complexity and computational overhead. Finally, features 5 and 6 are multiplied with feature 1 after sigmoid activation to obtain the final output Re-weight 2 $(H \times W \times C)$. 


\subsubsection{Do-Conv Convolution}

In general, the network depth is usually increased by combining linear convolutional layers and nonlinear network layers to increase the network expressiveness, since successive linear layers increase the overfitting phenomenon of the network and can be replaced by a linear layer. This paper replaces part of the traditional convolution in FPN + PANet with Do-Conv convolution, speeding up the network training and promoting the model convergence.

The operation of Do-Conv is shown in Figure 5, where * denotes conventional convolution and o denotes depth-wise convolution. In model training, the depth-wise convolution of weight $\mathbb{D}^{T} \in R^{(M \times N) \times C_{\text {in }}}$ and weight $\mathbb{W} \in R^{D_{m u} \times C_{\text {in }} \times C_{\text {out }}}$ are first computed to obtain

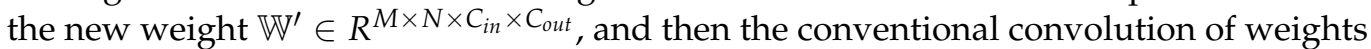
$\mathbb{W} /$ and input features $\mathbb{P}$ is calculated to get the final output $\mathbb{O}$, and it should be noted that $D_{m u l} \geq M \times N$. On the basis of traditional convolution, Do-Conv adds an additional depth-wise convolution, to form an over-parameterized convolution layer, which increases the number of parameters compared to conventional convolution. Although the number of parameters increases, the multi-layer linear operations used in over-parameterized convolution can be combined into a single-layer convolution operation during model inference, because both conventional convolution and depth-wise convolution are linear operations, thus speeding up the inference.

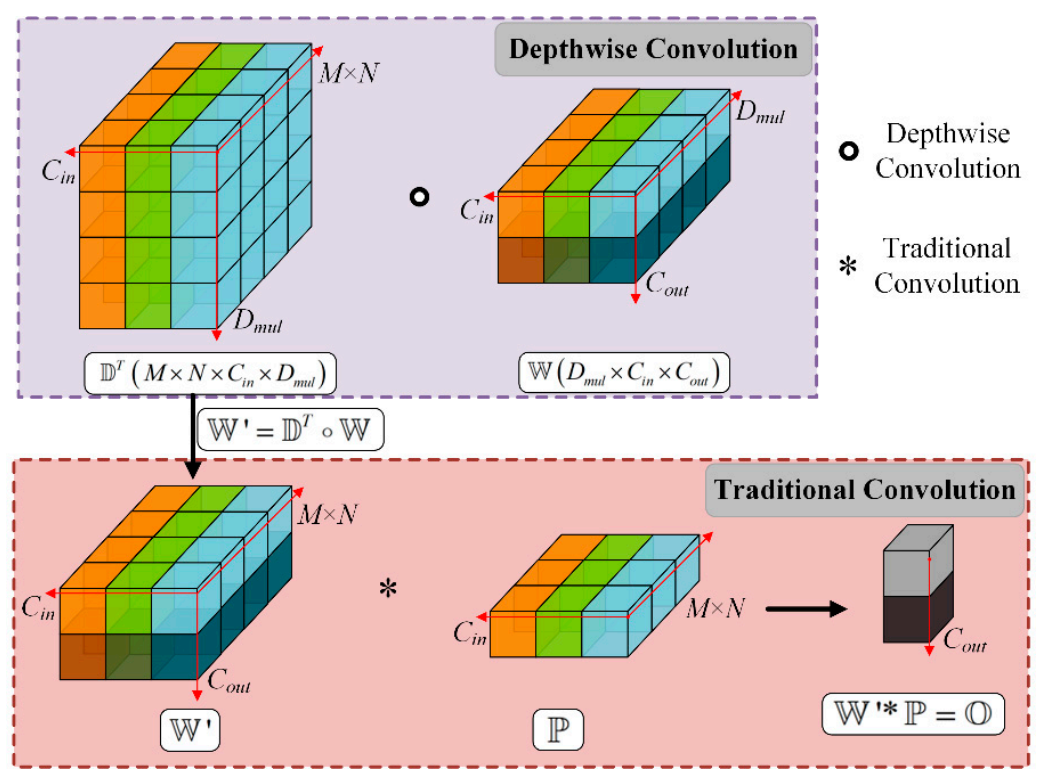

Figure 5. Do-Conv operation diagram.

\subsubsection{Focal Loss Function}

In the YOLOv4-LITE network training, it is necessary to first set a suitable intersection over union (IoU) threshold. When the IoU between the anchor box and all targets ground truth is less than the IoU threshold, this anchor box is regarded as a negative sample; and when the target centroid falls in a grid, the anchor box in the grid that has the maximum IoU with the target is a positive sample. In one-stage object detection, the loss function is dominated by many negative samples due to the imbalance between positive and negative samples during training, so the network cannot measure the prediction results. Therefore, this paper introduces a focal loss function to solve the problems of unbalanced positive and negative samples, and unbalanced simple samples and difficult samples. The focal loss function is calculated as follows:

$$
F L\left(p_{t}, y\right)=\left\{\begin{array}{c}
-\alpha\left(1-p_{t}\right)^{\gamma} \log _{a} p_{t}, y=1 \\
-(1-\alpha) p_{t}^{\gamma} \log _{a}\left(1-p_{t}\right), y=0
\end{array}\right.
$$


where $y$ is the category label and $p_{t}\left(p_{t} \in[0,1]\right)$ is the probability that the $t$-th sample is $y$. Considering the imbalance of positive and negative samples in the ginger image, most of the area is the background, and the number of positive samples (ginger and ginger shoots) is much lower than the negative samples (background). Thus, the paper adds a weighting factor $\alpha(\alpha \in[0.5,1))$ to the cross-entropy loss function so that a smaller number of positive samples take up more weight, and thus the model can learn more helpful information. On the other hand, considering the imbalance between simple and difficult samples, ginger samples are more facile to identify than ginger shoot samples. Focal loss combines the idea of OHNM, by adding a weighting factor $\left(1-p_{t}\right)^{\gamma}$ to the loss function, and $\gamma$ can be used to reduce the loss of simple samples by adjusting the variation range of weighting factor $\left(1-p_{t}\right)^{\gamma}$, and its value range is generally $[0,5]$. For instance, when $y_{t}=1$, the $p_{t}$ of the simple sample is close to 1 , so $\left(1-p_{t}\right)^{\gamma}$ is close to 0 . In contrast, $\left(1-p_{t}\right)^{\gamma}$ of the difficult sample is close to 1 . The above description implies that the addition of $\left(1-p_{t}\right)^{\gamma}$ makes the difficult samples have a more significant impact on the loss function. If $\gamma$ is too small, it will not increase the loss of difficult samples. On the contrary, if $\gamma$ is too large, it is not conducive to model training. In the end, $\gamma=2$ and $\alpha=0.75$.

\subsubsection{Identification Method of Ginger Shoot Orientation}

First, to discriminate the orientation of ginger shoots, the location of the ginger shoots and ginger is predicted using the ginger identification network. Second, this paper uses the area of the ginger shoot prediction box as the criterion to select ginger shoot and only selects the largest one to discriminate the orientation of ginger shoot. As shown in Figure 6, a right-angle coordinate system is established with the center point $O$ of the ginger prediction frame as the origin, the center point of the ginger prediction frame is $\mathrm{A}(\mathrm{d} x, \mathrm{~d} y)$, and the orientation angle of the ginger shoot is $\theta$, where " + " indicates counterclockwise rotation and "-" indicates clockwise rotation.

$$
\begin{gathered}
\theta \prime=\arctan \left(\frac{|d y|}{|d x|}\right) \\
\theta=\left\{\begin{array}{l}
+\theta \prime, \mathrm{d} x>0 \mathrm{~d} y \geq 0 \\
+\pi / 2, \mathrm{~d} x=0 \mathrm{~d} y>0 \\
+(\pi-\theta \prime), \mathrm{d} x<0 \mathrm{~d} y>0 \\
-(\pi-\theta \prime), \mathrm{d} x<0 \mathrm{~d} y \leq 0 \\
-\pi / 2, \mathrm{~d} x=0 \mathrm{~d} y<0 \\
-\theta \prime, \mathrm{d} x>0 \mathrm{~d} y<0
\end{array}\right.
\end{gathered}
$$

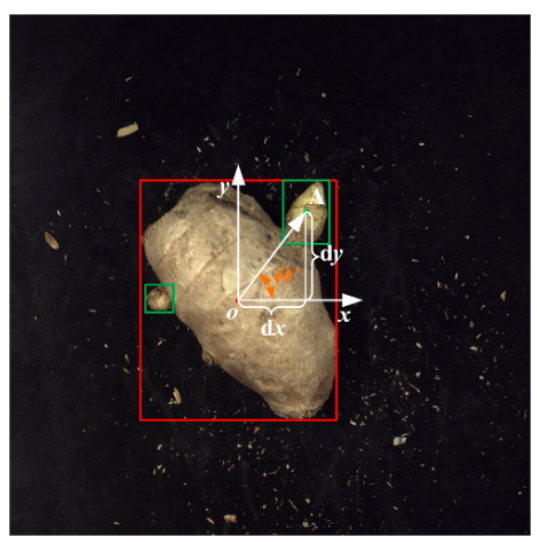

Figure 6. Calculation of ginger shoot orientation angle. 


\subsection{Method of Discriminating Ginger Shoot Orientation}

The paper uses precision $(P)$ and recall $(R)$ as evaluation criteria to assess the model performance. In addition, the F1 score can be used to equalize the precision and recall. They are defined as shown in Equations (5)-(7).

$$
\begin{gathered}
P=\frac{T P}{T P+F P} \times 100 \% \\
R=\frac{T P}{T P+F N} \times 100 \% \\
\text { F1 score }=\frac{2 P R}{P+R} \times 100 \%
\end{gathered}
$$

where true positive (TP) means that the prediction result and ground truth are both positive samples; false positive $(F P)$ indicates that the prediction result is positive and ground truth is negative; and false negative $(F N)$ means the prediction result is negative and ground truth is positive.

However, depending on different task requirements, precision and recall can be adjusted to various values during model testing by adjusting different confidence thresholds, and average precision $(A P)$, as the average of precision under different recalls can be used to measure the inherent model properties. In this study, since there are two categories, the ginger shoot and ginger seeds, mean average precision $(m A P)$ was adopted to measure the model performance. The equations of $A P$ and $m A P$ are as follows:

$$
\begin{gathered}
A P=\int_{0}^{1} P(R) d R \times 100 \% \\
m A P=\frac{1}{2} \sum_{m=0}^{1} \int_{0}^{1} P_{m}\left(R_{m}\right) d R_{m} \times 100 \%
\end{gathered}
$$

where $m$ is the number of categories and $R$ is the integral variable used to calculate the region's area under the $P-R$ curve. $A P_{50}$ is the $A P$ value when the IoU threshold is 0.5 ; therefore, $m A P_{50}$ is the average of $A P_{50}$ for all categories. Similarly, $m A P_{75}$ is the average of $A P_{75}$ of all categories, $m A P_{50: 95}$ is the average of $A P_{50: 95}$, and $A P_{50: 95}$ is the average of ten values of $A P_{50}, A P_{55}, A P_{60}, \ldots, A P_{95}$.

In addition, the model performance was measured using model size, Params, and giga Flops (GFlops) [50,51], where Params is the total number of parameters required to train the network, and GFlops is the amount of computation in the network. The lower the GFlops, the less computation and execution time needed for the model.

\section{Results and Discussion}

The experimental environment of the YOLOv4-LITE network during model training is shown in Table 2. In addition, the model optimizer was SGD (stochastic gradient descent), the momentum was 0.95 , the weight attenuation coefficient was $5 \times 10^{-3}$, the batch size was 16 , the trained epochs were 200, and the model weight was reserved once for every 10 epochs. At the beginning of the network training, the learning rate was increased linearly from 0 to $1 \times 10^{-4}$ in the first 20 epochs, to make the network converge to a better initial state quickly, and it was then reduced to $1 \times 10^{-6}$ by using the cosine annealing decay method; the formula and diagram of the learning rate are shown below.

$$
l r=\left\{\begin{array}{l}
\frac{l r_{\max }}{T_{\text {warm }}} t \quad t \leq T_{\text {warm }} \\
l r_{\text {min }}+\frac{1}{2}\left(l r_{\text {max }}-l r_{\text {min }}\right)\left(1+\cos \left(\frac{\mathrm{t}-T_{\text {warm }}}{T_{\text {total }}}\right)\right) \quad t>T_{\text {warm }}
\end{array}\right.
$$

where $t$ and $T_{\text {warm }}$ are the current epochs and warmup epochs, respectively; $l r_{\min }$ and $l r_{\max }$ represent the minimum and maximum values of the learning rate, respectively; $T_{\text {cur }}$ and $T_{\text {total }}$ represent the current and total epochs, respectively. 
Table 2. Experimental environment.

\begin{tabular}{cc}
\hline Configuration & Parameter \\
\hline CPU & Intel core 19-9900K \\
GPU & Nvidia GTX 2080Ti GPU \\
Operating system & Ubuntu 18.04 \\
Accelerated environment & CUDA 10.0 CUDNN 7.0 \\
Development environment & PyCharm professional edition \\
Library & Python 3.6, Pytorch1.5.1, Opencv4.2.0 \\
\hline
\end{tabular}

As shown in Figure 7b, the dimensions of the labeled boxes were clustered using the K-means algorithm before network training. K-means uses an IoU-based metric with the objective function of minimizing the distance between the labeled boxes and the clustered boxes, and resulted in nine clustered boxes, $(24,24),(33,42),(39,57),(51,64),(46,84)$, $(217,172),(287,204),(237,252)$, and $(329,285)$, which were then used to initialize the anchor boxes in the ginger recognition network. When network training, a multi-scale training method is used to improve the model generalization, which means randomly training the model with images of different sizes every 10 batches, while ensuring that the image edge length is a multiple of 32. Moreover, this paper also used mixed-precision training, based on single-precision and half-precision, to speed up the network training and reduce the GPU memory usage.

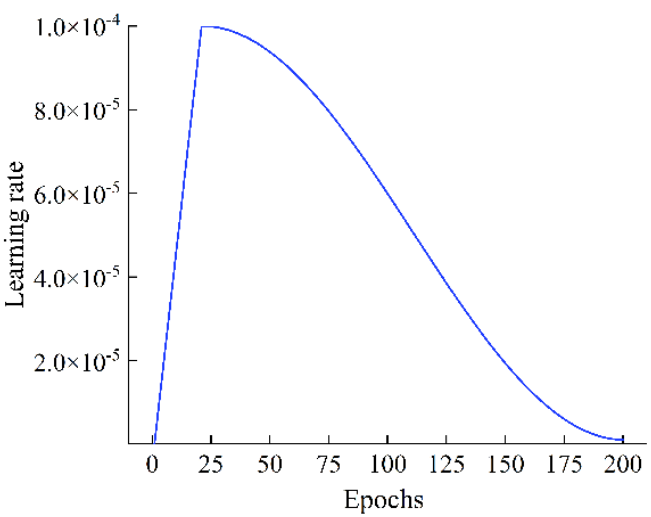

(a)

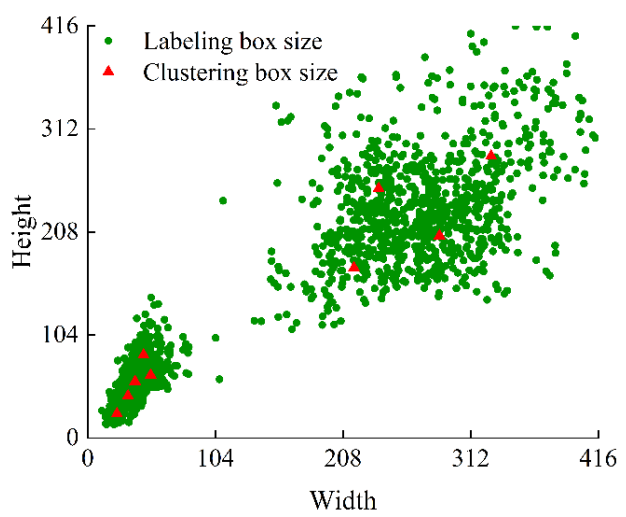

(b)

Figure 7. Schematic diagram of learning rate and labeling box. (a) Learning rate; (b) distribution of labelling boxes.

\subsection{Result Analysis}

As is well-known, the loss function evaluates a model by measuring the error between the predicted and the true values. Figure 8a shows the loss value change curve of YOLOv4LITE with a total training time of $3.5 \mathrm{~h}$. As seen in Figure 8a, the loss value dropped rapidly from 1441.33 to 3.71 in the first 40 epochs and then slowly oscillated down and stabilized as the epochs increased. Eventually, the loss value stabilized at around 1.80, and the model converged at the same time point. Figure $8 \mathrm{~b}$ shows the $P$ - $R$ curves for an IoU threshold of 0.5 . Both the $P-R$ curves for ginger shoots and ginger enclosed almost the entire parameter space, which indicated that the model had achieved a sufficient average precision. It can also be clearly seen from Figure $8 \mathrm{~b}$ that YOLOv4-LITE performed better in ginger recognition than the ginger shoots of smaller targets. 


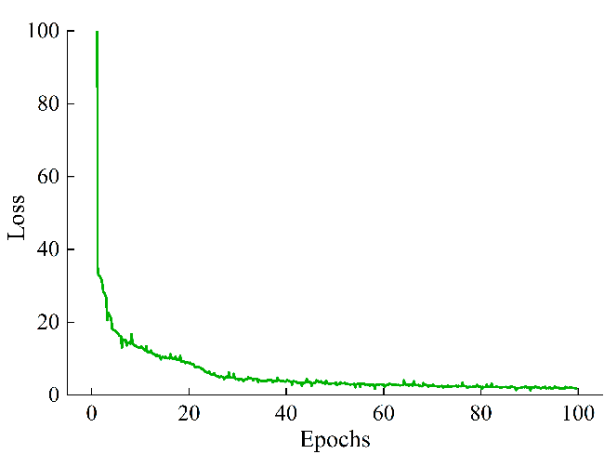

(a)

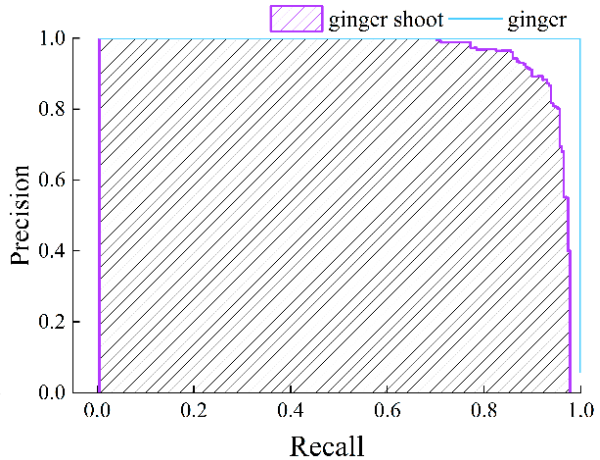

(b)

Figure 8. Loss curve and P-R curve comparison chart. (a) Loss curve; (b) P-R curve.

The test results of the validation set were analyzed, and the confidence threshold (conf-thresh) was taken as 0.5 , and the IoU threshold was taken as 0.5. The test results are shown in Table 3, and the number of the ground truth in the test set was 435 . The improved model had improvements in terms of precision, recall, and F1-score. Specifically, the precision increased by $0.49 \%$, recall increased by $1.15 \%$, and F1-score increased by $0.82 \%$. The analysis of $T P, F P$, and $F N$ in the test results revealed that the improved model performance mainly depended on the increase of $T P$ and the decrease of $F P$ and $F N$.

Table 3. Validation set test results after network improvement (conf-thresh $=0.5, \mathrm{IoU}=0.5$ ).

\begin{tabular}{ccccccc}
\hline Model & TP & FP & FN & P/\% & R/\% & F1-Score/\% \\
\hline YOLOv4 & 414 & 23 & 21 & 94.74 & 95.17 & 94.95 \\
YOLOv4-LITE & 419 & 21 & 16 & 95.23 & 96.32 & 95.77 \\
\hline
\end{tabular}

As shown in Figure 9a,b, this paper compared the original and manually labeled ginger seed images to better evaluate the recognition effect of the YOLOv4-LITE network. The final recognition results are as shown in Figure 9c, where the sizes of the test images are all $416 \times 416$ pixels, and the green and white rectangular boxes represent the predicted boxes for the ginger and ginger shoots, respectively. As can be seen in Figure 9, the ginger seed images were well recognized. The image above had only one ginger shoot, and the coordinates of the center points of the prediction boxes for ginger and shoot were $(204,207)$ and $(220,317)$, respectively. After the calculation of Equations (3) and (4), $\delta=-81.7^{\circ}$, the ginger seed was rotated clockwise by $81.7^{\circ}$ to ensure consistent ginger shoot orientation. The below image had two ginger shoots, as shown by the red arrows, only the ginger shoot with the larger prediction box was chosen. The coordinates of the center points of the prediction boxes for ginger and shoot are $(191,192)$ and $(270,238)$, respectively. After the calculation of Equations (3) and (4), $\delta=-30.2^{\circ}$, the ginger seed should be rotated clockwise by $30.2^{\circ}$.

Due to the very irregular shape of the ginger seeds and the fragility of the ginger shoots, we designed a ginger seed transport channel. After placing the ginger seeds on the channel, detection of the seeds and the orientation of the ginger shoots was achieved using an image acquisition device and a mobile terminal device. Next, an end-effector with vacuum suction cups was used to pick up the center of the ginger prediction box and adjust the orientation of the ginger shoot in real-time to ensure that the ginger shoots were facing the same direction. 


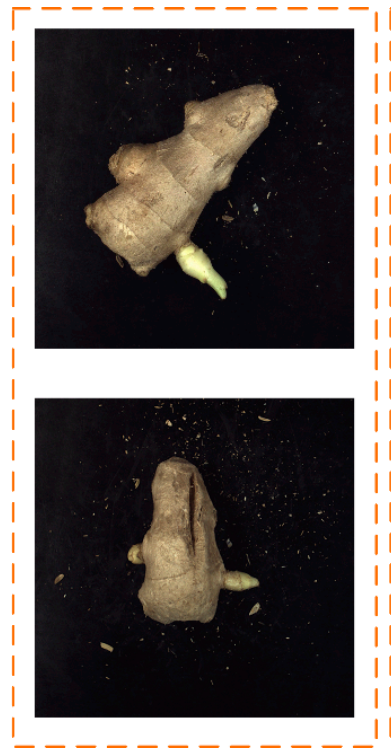

(a)

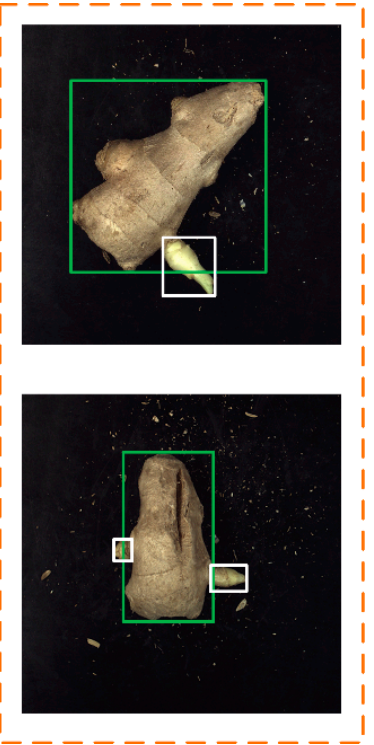

(b)
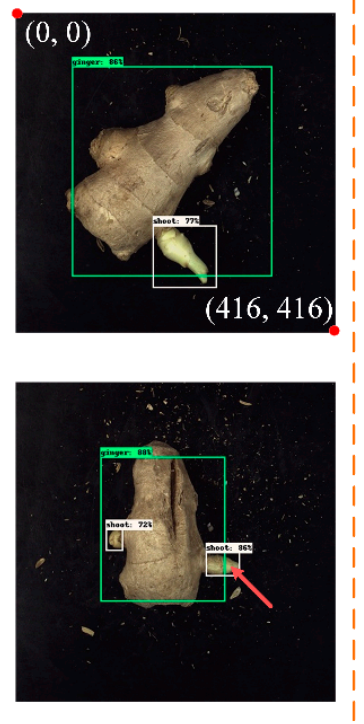

(c)

Figure 9. Ginger seed image recognition results. (a) Origin images, (b) labeled images, and (c) identified results.

\subsection{Discussion of the Improved Algorithm}

This paper conducted the following three comparison experiments [52] to demonstrate the contribution of the proposed improved refinements to the YOLOv4 network: comparison experiments after replacing the feature extraction network, experiments with different attention mechanisms, and comparison experiments after adding Do-Conv convolution.

\subsubsection{Performance Comparison of Feature Map Extraction Network}

The test results of the network are as shown in Figure 10, after replacing the original backbone network of YOLOv4 with the MobileNetv2 network. This strategy achieved good results, as $m A P_{50}$ was reduced by only $0.37 \%$. Moreover, compared to the original CSPDarknet53, the network computation was greatly reduced after using MobieNetv2 as the backbone network. As shown in Table 4, the model size, Params, and GFlops before and after the improvement of the backbone network were compared, and, notably, when calculating Params and GFlops, the network input images were of the same size, and $416 \times 416$ pixels was chosen for this paper. As can be seen from Table 4, YOLOv4-LITE using MobieNetv2 as the backbone network was much smaller than the original network, in terms of model size, Params, and GFlops; reducing these to $149.6 \mathrm{MB}, 15.95 \mathrm{M}$, and 21.14, respectively, which indicated that the improved network had a lower computational time and spatial complexity.

Table 4. Experimental results under different feature extraction networks.

\begin{tabular}{cccccc}
\hline $\begin{array}{c}\text { Backbone } \\
\text { Network }\end{array}$ & $\begin{array}{c}A P_{\mathbf{5 0}} / \% \\
(\text { Shoot) }\end{array}$ & $\begin{array}{c}A P_{\mathbf{5 0}} / \% \\
\text { (Ginger) }\end{array}$ & Size/MB & Params/M & GFlops \\
\hline CSPDarknet53 & 98.22 & 99.99 & 264.6 & 63.94 & 29.883 \\
MobileNetv2 & 97.45 & 99.99 & 115 & 47.99 & 8.741 \\
\hline
\end{tabular}




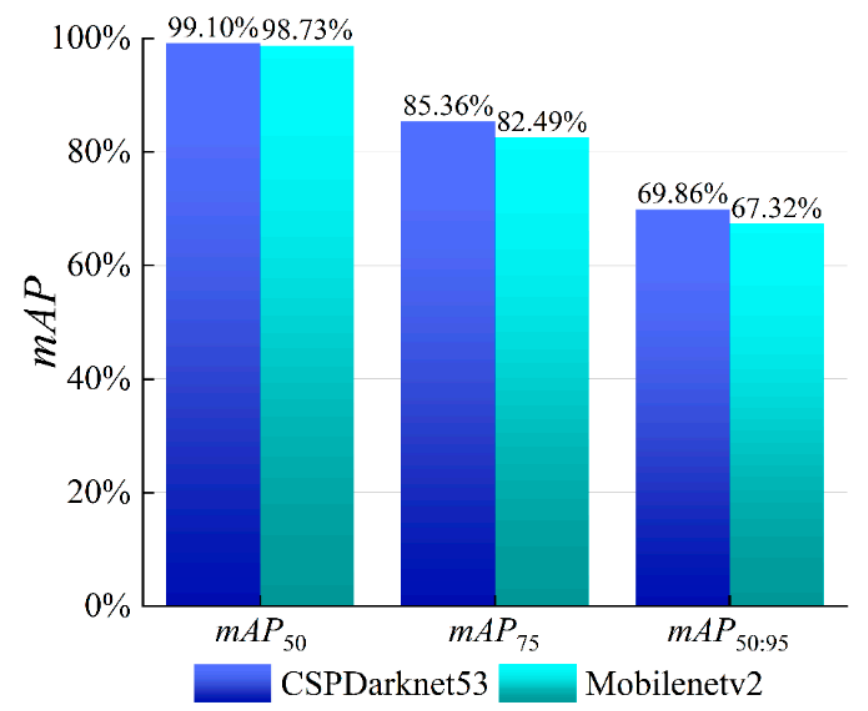

Figure 10. Contrast graphs of the improved backbone network.

\subsubsection{Different Attention Mechanisms Comparative Experiment}

In this paper, SE, CBAM, and CA attentions were inserted in the IRB module of MobileNetv2 to verify the effect of CA attention by comparing their test results. The test results shown in Figure 11 indicated that after using three kinds of attention modules, the $A P_{50}$ of ginger shoots increased by $2.64 \%, 3.52 \%$, and $5.95 \%$, respectively, while the $A P_{50}$ of ginger remained basically unchanged. It is worth mentioning that the $A P$ of ginger shoots improved most significantly after using $C A$ attention, with $A P_{50}$ increasing from $91.5 \%$ to $97.45 \%$ and $A P_{50: 95}$ rising from $41.63 \%$ to $52.32 \%$, indicating that the addition of CA attention to YOLOv4-LITE effectively improved the detection accuracy of ginger shoots.

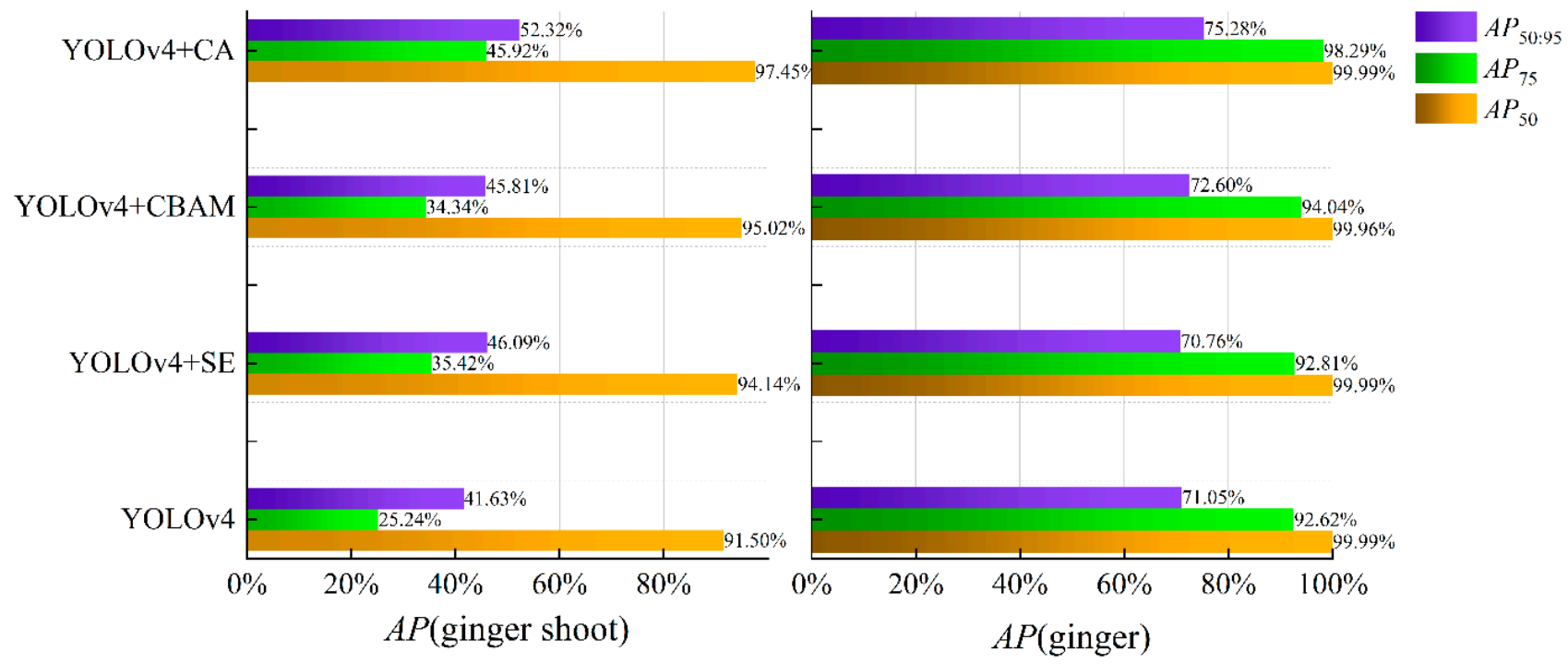

Figure 11. Contrast graph of different attention modules.

\subsubsection{Analysis of Do-Conv Convolution}

This paper conducted a comparison experiment with Do-Conv instead of the conventional convolution in the FPN + PANet structure, to study the effect of Do-Conv convolution on the ginger seed recognition network. The loss curves of the YOLOv4-LITE network are shown in Figure 12, which indicate that the network had a faster convergence rate after using Do-Conv convolution in the training process. Moreover, the network test results 
after using Do-Conv are shown in Table 5, which show that the $A P_{50}$ of ginger shoots improved by $2.18 \%$. Furthermore, although the Do-Conv convolution layer added an extra depth-wise convolution to the conventional convolution, it did not increase the Params and GFlops. The reason for this was as follows: during the model training, $\mathbb{D}$ and $\mathbb{W}$ were folded into $\mathbb{W}^{\prime}\left(\mathbb{W}^{\prime}=\mathbb{D}^{T} \circ \mathbb{W}\right)$ with the same shape as the conventional convolution kernel that they replaced, so the Params and GFlops of the model did not change in the inference.

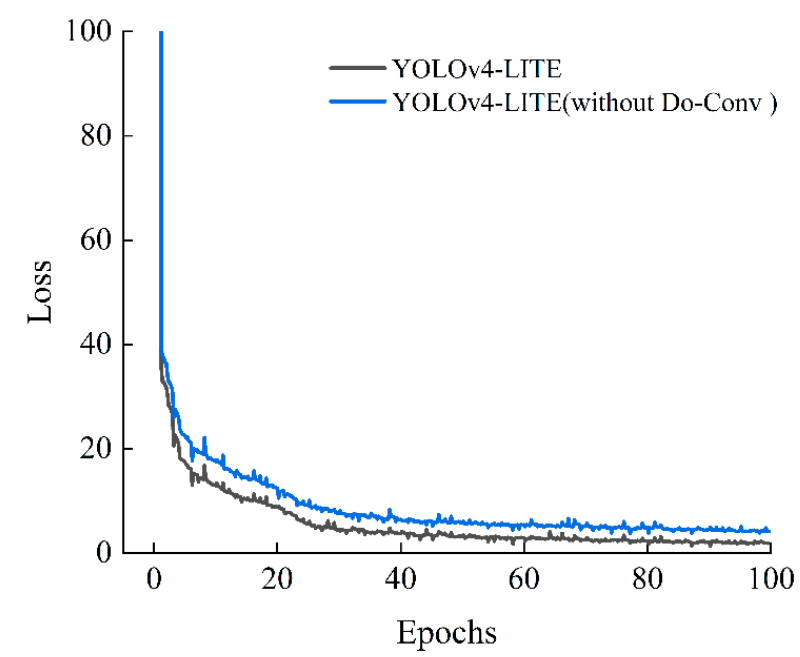

Figure 12. Loss value change curve.

Table 5. Testing set experiment results using Do-Conv.

\begin{tabular}{|c|c|c|c|c|c|}
\hline Model & $\begin{array}{c}A P_{50} / \% \text { (Ginger } \\
\text { Shoot) }\end{array}$ & $A P_{50} / \%$ (Shoot) & $m A P / \%$ & Params/M & GFlops \\
\hline YOLOv4-LITE & 97.45 & 99.99 & 98.72 & 47.99 & 8.741 \\
\hline $\begin{array}{c}\text { YOLOv4-LITE } \\
\text { (without Do-Conv) }\end{array}$ & 95.27 & 99.99 & 97.63 & 47.99 & 8.741 \\
\hline
\end{tabular}

\subsection{Performance Comparison of the Overall Algorithm}

Figure 13 shows the $m A P$ of the various improved algorithms. Compared with YOLOv3 and YOLOv3-tiny [53], YOLOv4 had a better target detection performance, with a $m A P_{50}$ of $99.1 \%$, which was $1 \%$ and $1.8 \%$ higher than the others, respectively. When replacing the backbone network with MobileNetv3 [54], Ghost-Net [55], and MobileNetv2, without using other improved strategies, the network detection performance dropped dramatically, with a $m A P_{50}$ of only $92.75 \%, 93.13 \%$, and $93.54 \%$. After using CA attention or Do-Conv based on MobileNetv2, the $m A P_{50}$ reached $97.63 \%$ and $97.25 \%$, respectively; while, after using CA attention and Do-Conv at the same time, $m A P_{50}$ reached $98.73 \%$. In summary, this study effectively enhanced the network performance through the series of improvements mentioned above.

For the recognition of ginger seed images, we tried to use the traditional color difference segmentation method to segment ginger shoots and recorded the color components of ginger seed images using RGB (red green blue) and HSV (hue saturation value). It was found that the $\mathrm{H}$ component is less influenced by the illumination and can achieve the segmentation of ginger shoots. However, the ginger seeds were treated with drugs resulting in unstable color characteristics of the ginger shoots, thus making the error rate of identification high; therefore, the color difference segmentation method is not very reliable for the segmentation of ginger shoots. 


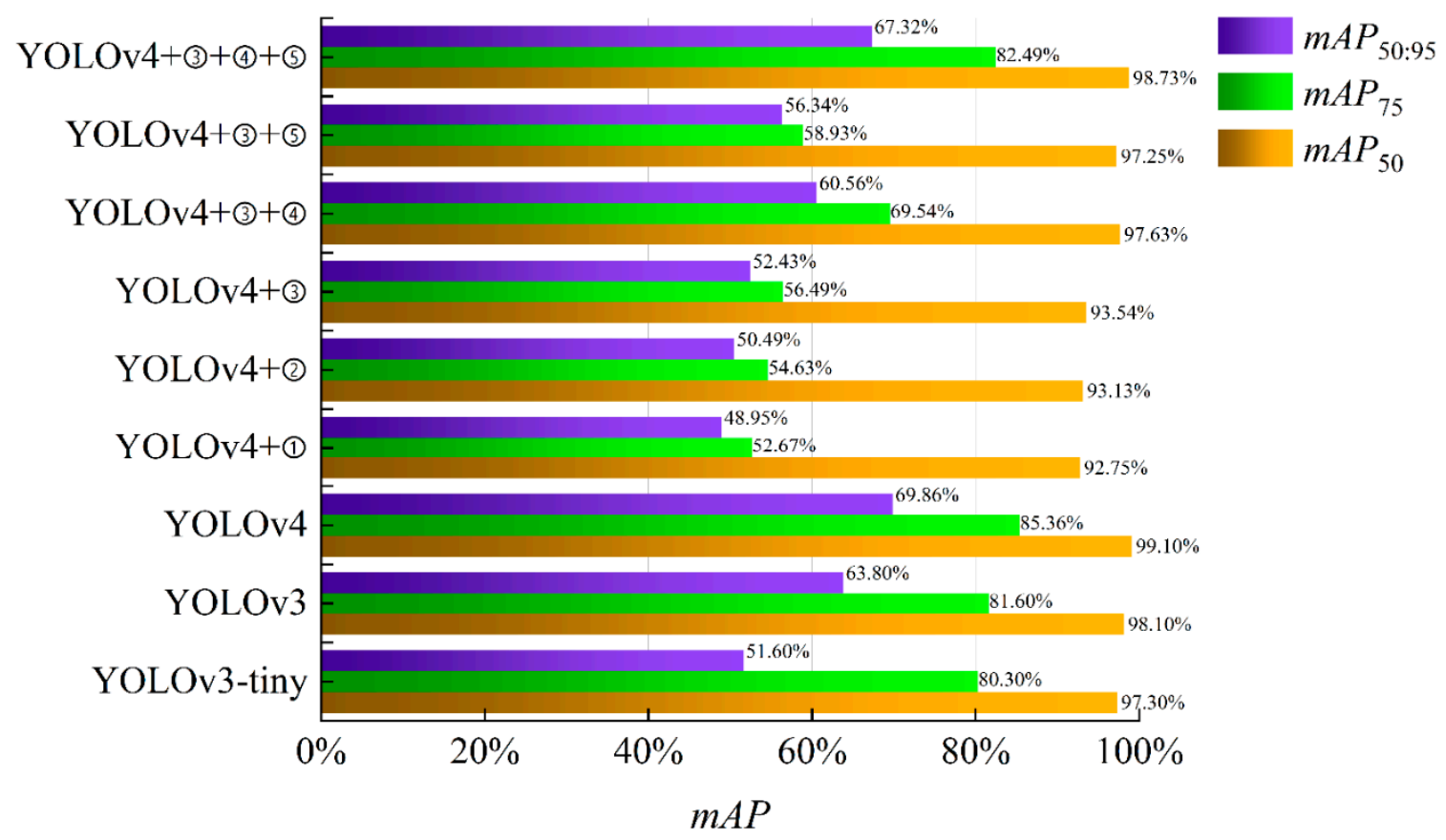

(1)MobileNetv3. (2) Ghost-Net. (3) MobileNetv2. (4)CA attention. (5Do-Conv.

Figure 13. Contrast graph of the various improved algorithms.

Unlike the traditional color difference segmentation method, Hou et al. proposed a fast recognition method for ginger shoots based on YOLOv3. The method avoided the manual design of feature extractors and had good robustness, and the AP of ginger shoots reached $98.2 \%$. However, it only identified ginger shoots and did not identify ginger, resulting in a complex calculation process for ginger shoot orientation. In addition, its backbone network was complex and contained a large number of redundant parameters, making it difficult to deploy on ginger seeders and also restricting the development of automated ginger seeders to some extent.

Based on the constructed ginger seed dataset, the backbone network of YOLOv4 was replaced by MobileNetv2, which greatly reduced the network parameters and computational effort. Meanwhile, CA attention and Do-Conv convolution were added to the backbone network to improve the detection of ginger shoots and the convergence speed of the model. The experimental results showed that $m A P_{50}$ reached $98.72 \%$ and $m A P_{75}$ reached $82.46 \%$.

\section{Conclusions}

To achieve ginger seed detection and ginger shoot orientation discrimination, this paper introduced an improved YOLOv4-LITE network to detect ginger shoots and ginger in ginger seed images and then discriminated ginger shoot orientation by calculating the position of the largest ginger shoot relative to the ginger. First, this paper replaced the original CSPDarknet53 backbone network with MobileNetv2, which significantly reduced the network parameters and computation; thus, facilitating migration of the network to a mobile terminal. Second, a coordinate attention mechanism was added into the backbone network to improve the detection of ginger shoots. Third, Do-Conv was adopted to replace some traditional convolutions, thus improving the model convergence speed. Finally, the paper also used focal loss to solve the imbalance between positive and negative samples and the imbalance between simple and difficult samples in the ginger dataset.

The experimental results showed that the $m A P_{50}$ of the proposed improved YOLOv4LITE network reached $98.73 \%$. Compared with the original YOLOv4, its Params and GFlops decreased by $15.95 \mathrm{M}$ and 21.14 , respectively, while the $m A P_{50}$ was only reduced by 
$0.37 \%$. This indicates that using the improved backbone network and Do-Conv convolution is effective for improving the performance of the ginger seed recognition network.

Author Contributions: All authors contributed to the research. Conceptualization, L.F. and Y.W.; methodology, L.F. and Y.W.; software, L.F.; validation, L.F. and Y.L.; formal analysis, L.F. and Y.W.; investigation, L.F., H.G., H.Z., X.W. and J.H.; resources, J.H. and Y.W.; data curation, L.F.; writingoriginal draft preparation, L.F. and J.H.; writing-review and editing, J.H. and R.X.; visualization, L.F.; supervision, J.H.; project administration, J.H.; funding acquisition, J.H. All authors have read and agreed to the published version of the manuscript.

Funding: This work was supported by China Agriculture Research System of MOF and MARA (Grant numbers: CARS-24-D-01), the Shandong Agricultural Major Applied Technology Innovation Project (Grant numbers: SD2019NJ004), and the Shandong Modern Agricultural Industry Technology System Vegetable Industry Innovation Team Project (Grant numbers: SDAIT-05).

Data Availability Statement: Not applicable.

Conflicts of Interest: The authors declare no conflict of interest.

\section{References}

1. Retana-Cordero, M.; Fisher, P.R.; Gómez, C. Modeling the Effect of Temperature on Ginger and Turmeric Rhizome Sprouting. Agronomy 2021, 11, 1931. [CrossRef]

2. Sang, S.; Snook, H.D.; Tareq, F.S.; Fasina, Y. Precision Research on Ginger: The Type of Ginger Matters. J. Agric. Food Chem. 2020, 68, 8517-8523. [CrossRef]

3. Liu, S.; Chen, M.; He, T.; Ren, C. Review of China's ginger market in 2018 and market outlook in 2019. China Veget. 2019, 2, 1-4.

4. Zhang, K.; Jiang, H.; Liu, P.; Zhang, Y. Prediction of ginger planting area based on GM(1,N) model. J. Chin. Agric. Mech. 2020, 41, 139-143.

5. Tao, W. Technical specifications for the safe production of ginger. In Laiwu Ginger, 1st ed.; China Agricultural Science and Technology Press: Beijing, China, 2010; pp. 78-79.

6. Mahender, B.; Reddy, P.S.S.; Sivaram, G.T.; Balakrishna, M.; Prathap, B. Effect of seed rhizome size and plant spacing on growth, yield and quality of ginger (Zingiber officinale rosc.) under coconut cropping system. Plant Arch. 2015, 15, 769-774.

7. Liu, J. Biological properties of ginger. In Laiwu Ginger, 1st ed.; China Agricultural Science and Technology Press: Beijing, China, 2013; pp. 76-77.

8. Hordofa, T.S.; Tolossa, T.T. Cultivation and postharvest handling practices affecting yield and quality of major spices crops in Ethiopia: A review. Cogent Food Agric. 2020, 6. [CrossRef]

9. Ren, S.; Li, C. Analysis of the current situation and development of the ginger industry in China. China Veget. $2021,8,8-11$.

10. Xiong, X.; Duan, L.; Liu, L.; Tu, H.; Yang, P.; Wu, D.; Chen, G.; Xiong, L.; Yang, W.; Liu, Q. Panicle-SEG: A robust image segmentation method for rice panicles in the field based on deep learning and superpixel optimization. Plant Methods 2017, 13, 1-15. [CrossRef]

11. Li, H.; Wang, G.; Dong, Z.; Wei, X.; Wu, M.; Song, H.; Amankwah, S.O.Y. Identifying Cotton Fields from Remote Sensing Images Using Multiple Deep Learning Networks. Agronomy 2021, 11, 174. [CrossRef]

12. Gahrouei, O.; McNairn, H.; Hosseini, M.; Homayouni, S. Estimation of Crop Biomass and Leaf Area Index from Multitemporal and Multispectral Imagery Using Machine Learning Approaches. Can. J. Remote Sens. 2020, 46, 84-99. [CrossRef]

13. Bahrami, H.; Homayouni, S.; Safari, A.; Mirzaei, S.; Mahdianpari, M.; Reisi-Gahrouei, O. Deep Learning-Based Estimation of Crop Biophysical Parameters Using Multi-Source and Multi-Temporal Remote Sensing Observations. Agronomy 2021, 11, 1363. [CrossRef]

14. Wang, C.; Xiao, Z. Lychee Surface Defect Detection Based on Deep Convolutional Neural Networks with GAN-Based Data Augmentation. Agronomy 2021, 11, 1500. [CrossRef]

15. Guo, Q.; Wang, C.; Xiao, D.; Huang, Q. An Enhanced Insect Pest Counter Based on Saliency Map and Improved Non-Maximum Suppression. Insects 2021, 12, 705. [CrossRef]

16. Parvathi, S.; Selvi, S.T. Detection of maturity stages of coconuts in complex background using Faster R-CNN model. Biosyst. Eng. 2021, 202, 119-132. [CrossRef]

17. Ren, S.; He, K.; Girshick, R.; Sun, J. Faster R-CNN: Towards real-time object detection with region proposal networks. IEEE Trans. Pattern Anal. Mach. Intell. 2017, 39, 1137-1149. [CrossRef] [PubMed]

18. Jiao, L.; Dong, S.; Zhang, S.; Xie, C.; Wang, H. AF-RCNN: An anchor-free convolutional neural network for multi-categories agricultural pest detection. Comput. Electron. Agric. 2020, 174, 105522. [CrossRef]

19. He, K.; Gkioxari, G.; Dollár, P.; Girshick, R. Mask R-CNN. In Proceedings of the 2017 IEEE International Conference on Computer Vision (ICCV), Venice, Italy, 22-29 October 2017.

20. Ammar, A.; Koubaa, A.; Benjdira, B. Deep-Learning-Based Automated Palm Tree Counting and Geolocation in Large Farms from Aerial Geotagged Images. Agronomy 2021, 11, 1458. [CrossRef] 
21. Kuznetsova, A.; Maleva, T.; Soloviev, V. Using YOLOv3 Algorithm with Pre- and Post-Processing for Apple Detection in Fruit-Harvesting Robot. Agronomy 2020, 10, 1016. [CrossRef]

22. Redmon, J.; Divvala, S.; Girshick, R.; Farhadi, A. You only look once: Unified, real-time object detection. In Proceedings of the 2016 IEEE Conference on Computer Vision and Pattern Recognition (CVPR), Las Vegas, NV, USA, 27-30 June 2016.

23. Suo, R.; Gao, F.; Zhou, Z.; Fu, L.; Song, Z.; Dhupia, J.; Li, R.; Cui, Y. Improved multi-classes kiwifruit detection in orchard to avoid collisions during robotic picking. Comput. Electron. Agric. 2021, 182, 106052. [CrossRef]

24. Wu, D.; Lv, S.; Jiang, M.; Song, H. Using channel pruning-based YOLO v4 deep learning algorithm for the real-time and accurate detection of apple flowers in natural environments. Comput. Electron. Agric. 2020, 178, 105742. [CrossRef]

25. Liu, W.; Anguelov, D.; Erhan, D.; Szegedy, C.; Reed, E.S.; Fu, C.-Y.; Berg, A.C. SSD: Single shot multiBox detector. In Proceedings of the 14th European Conference on Computer Vision (ECCV), Amsterdam, The Netherlands, 11-14 October 2016.

26. Lin, T.; Goyal, P.; Girshick, R.; He, K.; Dollár, P. Focal Loss for Dense Object Detection. IEEE Trans. Pattern Anal. Mach. Intell. 2020, 42, 318-327. [CrossRef]

27. Koirala, A.; Walsh, K.B.; Wang, Z.; Anderson, N. Deep Learning for Mango (Mangifera indica) Panicle Stage Classification. Agronomy 2020, 10, 143. [CrossRef]

28. Hou, J.; Fang, L.; Wu, Y.; Li, Y.; Xi, R. Rapid recognition and orientation determination of ginger shoots with deep learning. Trans. Chin. Soc. Agric. Eng. 2021, 37, 213-222.

29. Cai, K.; Miao, X.; Wang, W.; Pang, H.; Liu, Y.; Song, J. A modified YOLOv3 model for fish detection based on MobileNetv1 as backbone. Aquac. Eng. 2020, 91, 102117. [CrossRef]

30. Yu, Y.; Zhang, K.; Liu, L.H.; Yang, L.; Zhang, D.X. Real-time visual localization of the picking points for a ridge-planting strawberry harvesting robot. IEEE Access 2020, 8, 116556-116568. [CrossRef]

31. Lui, S.; Lu, S.; Li, Z.; Hong, T.; Xue, Y.; Wu, B. Orange recognition method using improved YOLOv3-LITE lightweight neural network. Trans. Chin. Soc. Agric. Eng. 2019, 35, 205-214.

32. Ying, B.; Xu, Y.; Zhang, S.; Shi, Y.; Liu, L. Weed detection in images of carrot fields based on improved YOLO v4. Trait. Signal 2021, 38, 341-348. [CrossRef]

33. Shi, R.; Li, T.; Yamaguchi, Y. An attribution-based pruning method for real-time mango detection with YOLO network. Comput. Electron. Agric. 2020, 169, 105214. [CrossRef]

34. Bazame, H.C.; Molin, J.P.; Althoff, D.; Martello, M. Detection, classification, and mapping of coffee fruits during harvest with computer vision. Comput. Electron. Agric. 2021, 183, 106066. [CrossRef]

35. Buzzy, M.; Thesma, V.; Davoodi, M.; Mohammadpour Velni, J. Real-Time Plant Leaf Counting Using Deep Object Detection Networks. Sensors 2020, 20, 6896. [CrossRef]

36. Parico, A.I.B.; Ahamed, T. Real Time Pear Fruit Detection and Counting Using YOLOv4 Models and Deep SORT. Sensors 2021, 21, 4803. [CrossRef]

37. Hu, J.; Shen, L.; Albanie, S.; Sun, G.; Wu, E. Squeeze-and-Excitation Networks. IEEE Trans. Pattern Anal. Mach. Intell. 2020, 42, 2011-2023. [CrossRef]

38. Woo, S.; Park, J.; Lee, J.-Y.; Kweon, I.S. CBAM: Convolutional block attention module. In Proceedings of the 15th European Conference on Computer Vision (ECCV), Munich, Germany, 8-14 September 2018.

39. Kang, J.; Liu, L.; Zhang, F.; Shen, C.; Wang, N.; Shao, L. Semantic segmentation model of cotton roots in-situ image based on attention mechanism. Comput. Electron. Agric. 2021, 189, 106370. [CrossRef]

40. Xu, X.; Li, W.; Duan, Q. Transfer learning and SE-ResNet152 networks-based for small-scale unbalanced fish species identification. Comput. Electron. Agric. 2021, 180, 105878. [CrossRef]

41. Yang, B.; Gao, Z.; Gao, Y.; Zhu, Y. Rapid Detection and Counting of Wheat Ears in the Field Using YOLOv4 with Attention Module. Agronomy 2021, 11, 1202. [CrossRef]

42. Tang, Z.; Yang, J.; Li, Z.; Qi, F. Grape disease image classification based on lightweight convolution neural networks and channelwise attention. Comput. Electron. Agric. 2020, 178, 105735. [CrossRef]

43. Li, G.; Huang, X.; Ai, J.; Yi, Z.; Xie, W. Lemon-YOLO: An efficient object detection method for lemons in the natural environment IET Image Process. 2021, 15, 1998-2009. [CrossRef]

44. Liu, Z.; Wang, S. Broken corn detection based on an adjusted YOLO with focal loss. IEEE Access 2019, 7, 68281-68289. [CrossRef]

45. Li, Z.; Li, Y.; Yang, Y.; Guo, R.; Yang, J.; Yue, J.; Wang, Y. A high-precision detection method of hydroponic lettuce seedlings status based on improved Faster RCNN. Comput. Electron. Agric. 2021, 182, 106054. [CrossRef]

46. Sandler, M.; Howard, A.; Zhu, M.; Zhmoginov, A.; Chen, L. MobileNetV2: Inverted residuals and linear bottlenecks. In Proceedings of the 2018 IEEE Conference on Computer Vision and Pattern Recognition (CVPR), Salt Lake City, UT, USA, 18-22 June 2018.

47. Hou, Q.; Zhou, D.; Feng, J. Coordinate Attention for Efficient Mobile Network Design. In Proceedings of the 2021 IEEE Conference on Computer Vision and Pattern Recognition (CVPR), Nashville, TN, USA, 19-25 June 2021.

48. Cao, J.; Li, Y.; Sun, M.; Chen, Y.; Lischinski, D.; Cohen-Or, D.; Chen, B.; Tu, C. DO-Conv: Depthwise over-parameterized convolutional layer. arXiv 2020, arXiv:2006.12030.

49. Liu, S.; Qi, L.; Qin, H.; Shi, J.; Jia, J. Path aggregation network for instance segmentation. In Proceedings of the 2018 IEEE Conference on Computer Vision and Pattern Recognition (CVPR), Salt Lake City, UT, USA, 18-22 June 2018. 
50. Lee, D.; Kim, J.; Jung, K. Improving Object Detection Quality by Incorporating Global Contexts via Self-Attention. Electronics 2021, 10, 90. [CrossRef]

51. Li, Z.; Yang, Y.; Li, Y.; Guo, R.; Yang, J.; Yue, J. A solanaceae disease recognition model based on SE-Inception. Comput. Electron. Agric. 2020, 178, 105792. [CrossRef]

52. Ma, L.; Xie, W.; Huang, H. Convolutional neural network based obstacle detection for unmanned surface vehicle. Math. Biosci. Eng. 2020, 17, 845-861. [CrossRef] [PubMed]

53. Wu, D.; Wu, Q.; Yin, X.; Jian, B.; Wang, H.; He, D.; Song, H. Lameness detection of dairy cows based on the YOLOv3 deep learning algorithm and a relative step size characteristic vector. Biosyst. Eng. 2020, 189, 150-163. [CrossRef]

54. Zhang, X.; Kang, X.; Feng, N.; Liu, G. Automatic recognition of dairy cow mastitis from thermal images by a deep learning detector. Comput. Electron. Agric. 2020, 178, 105754.

55. Han, K.; Wang, Y.; Tian, Q.; Guo, J.; Xu, C.; Xu, C. GhostNet: More features from cheap operations. In Proceedings of the 2020 IEEE/CVF Conference on Computer Vision and Pattern Recognition (CVPR), Seattle, WA, USA, 13-19 June 2020. 